\title{
Front Matter: Volume 6891
}

, "Front Matter: Volume 6891," Proc. SPIE 6891, Organic Photonic Materials and Devices X, 689101 (11 March 2008); doi: 10.1117/12.791599

SPIE Event: Integrated Optoelectronic Devices 2008, 2008, San Jose, California, SPIE. United States 


\section{PROCEEDINGS OF SPIE}

\section{Organic Photonic Materials and Devices $X$}

Robert L. Nelson

Francois Kajzar

Toshikuni Kaino

Editors

22-24 January 2008

San Jose, California, USA

Sponsored and Published by

SPIE

Volume 6891 
The papers included in this volume were part of the technical conference cited on the cover and title page. Papers were selected and subject to review by the editors and conference program committee. Some conference presentations may not be available for publication. The papers published in these proceedings reflect the work and thoughts of the authors and are published herein as submitted. The publisher is not responsible for the validity of the information or for any outcomes resulting from reliance thereon.

Please use the following format to cite material from this book:

Author(s), "Title of Paper," in Organic Photonic Materials and Devices X, edited by Robert L. Nelson, Francois Kajzar, Toshikuni Kaino, Proceedings of SPIE Vol. 6891 (SPIE, Bellingham, WA, 2008) Article CID Number.

ISSN 0277-786X

ISBN 9780819470669

Published by

SPIE

P.O. Box 10, Bellingham, Washington 98227-0010 USA

Telephone +1 3606763290 (Pacific Time) · Fax +1 3606471445

SPIE.org

Copyright (c) 2008, Society of Photo-Optical Instrumentation Engineers

Copying of material in this book for internal or personal use, or for the internal or personal use of specific clients, beyond the fair use provisions granted by the U.S. Copyright Law is authorized by SPIE subject to payment of copying fees. The Transactional Reporting Service base fee for this volume is $\$ 18.00$ per article (or portion thereof), which should be paid directly to the Copyright Clearance Center (CCC), 222 Rosewood Drive, Danvers, MA 01923. Payment may also be made electronically through CCC Online at copyright.com. Other copying for republication, resale, advertising or promotion, or any form of systematic or multiple reproduction of any material in this book is prohibited except with permission in writing from the publisher. The CCC fee code is 0277-786X/08/\$18.00.

Printed in the United States of America.

Publication of record for individual papers is online in the SPIE Digital Library.

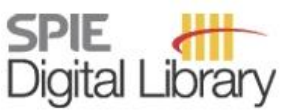

SPIEDigitalLibrary.org

Paper Numbering: Proceedings of SPIE follow an e-First publication model, with papers published first online and then in print and on CD-ROM. Papers are published as they are submitted and meet publication criteria. A unique, consistent, permanent citation identifier (CID) number is assigned to each article at the time of the first publication. Utilization of CIDs allows articles to be fully citable as soon they are published online, and connects the same identifier to all online, print, and electronic versions of the publication. SPIE uses a six-digit CID article numbering system in which:

- The first four digits correspond to the SPIE volume number.

- The last two digits indicate publication order within the volume using a Base 36 numbering system employing both numerals and letters. These two-number sets start with 00, 01, 02, 03, 04, 05, $06,07,08,09,0 \mathrm{~A}, 0 \mathrm{~B} \ldots \mathrm{OZ}$, followed by 10-1Z, 20-2Z, etc.

The CID number appears on each page of the manuscript. The complete citation is used on the first page, and an abbreviated version on subsequent pages. Numbers in the index correspond to the last two digits of the six-digit CID number. 


\section{Contents}

vii Conference Committee

\section{SESSION 1 MULTIPHOTON ABSORPTION}

689103 Near IR two photon absorption of cyanines dyes: application to optical power limiting at telecommunication wavelengths (Invited Paper) [6891-02]

P.-A. Bouit, Lab. de Chimie, ENS-Lyon, Univ. de Lyon (France); G. Wetzel, P. Feneyrou, Thales Research \& Technology (France); Y. Bretonnière, Lab. de Chimie, ENS-Lyon, Univ. de Lyon (France); K. Kamada, Photonics Research Institute, AIST (Japan); O. Maury, C. Andraud, Lab. de Chimie, ENS-Lyon, Univ. de Lyon (France)

689104 Two-photon absorption in cross-shaped chromophores with phenylene-vinylene backbones [6891-03]

M. Rumi, Georgia Institute of Technology (USA); S. J. K. Pond, The Univ. of Arizona (USA);

Q. Zhang, Georgia Institute of Technology (USA) and The Univ. of Arizona (USA); M. Bishop,

Y. Zhang, The Univ. of Arizona (USA); S. Barlow, Georgia Institute of Technology (USA) and

The Univ. of Arizona (USA); S. R. Marder, J. W. Perry, Georgia Institute of Technology (USA)

689105 Two-photon absorption standards in the 550-1600 nm excitation range: establishing a correction curve for accurate cross section calibration [6891-04]

N. S. Makarov, M. Drobizhev, A. Rebane, Montana State Univ., Bozeman (USA)

\section{SESSION 2 NONLINEAR OPTICS}

689109 Photostability of nonlinear optical polymers [6891-08]

S. G. Raymond, H. Wang, G. V. M. Williams, A. J. Kay, Industrial Research Ltd. (New Zealand)

\section{SESSION 3 MATERIALS}

6891 OA Monte Carlo kinetic study of chromophore distribution in poled guest-host system (Invited Paper) [6891-16]

A. C. Mitus, G. Pawlik, Wroclaw Univ. of Technology (Poland); I. Rau, Univ. of Bucharest (Romania); F. Kajzar, Lab. Poma, CNRS, Univ. d'Angers (France)

6891 OD Optical properties of calixarene polymers [6891-12]

T. Kim, K. Komatsu, O. Sugihara, T. Kaino, Tohoku Univ. (Japan); H. Kudo, T. Nishikubo, Kanagawa Univ. (Japan) 
6891 OF $90^{\circ}$-bent with $\mathrm{R}=1 \mathrm{~mm}$ optical fiber technique for optical interconnection (Invited Paper) [6891-46]

M. Morimoto, K. Suematsu, R. Sugizaki, K. Takahashi, H. Nasu, Furukawa Electric Co., Ltd. (Japan)

$68910 G$ Development of an optically gated optical switch using organic dye: applied to local telecommunication technology (Invited Paper) [6891-15]

T. Hiraga, I. Ueno, National Institute of Advanced Industrial Science and Technology (Japan); H. Nagaeda, N. Shiga, Trimatiz, Ltd. (Japan); H. Watanabe, S. Futaki, Inter Energy Co., Ltd. (Japan); N. Tanaka, Dainichiseika Color \& Chemicals Manufacturing Co., Ltd. (Japan)

$6891 \mathrm{OH} \quad$ Optical information recording in biopolymer-based material [6891-09]

J. Mysliwiec, A. Kochalska, A. Miniewicz, Wroclaw Univ. of Technology (Poland)

\section{SESSION 5 BIO- AND NANOMATERIALS}

6891 OM Thermo-optic coefficients of hybrid polymer with metal oxide nanoparticles [6891-21] Y. Kurata, Tohoku Univ. (Japan); K. Komatsu, Sendai National College of Technology (Japan); O. Sugihara, T. Kaino, Tohoku Univ. (Japan); N. Kambe, NanoGram Corp. (USA)

\section{SESSION 6 OPTICAL WAVEGUIDE}

689100 Recent progress on polymer optical waveguides (Invited Paper) [6891-23]

J. Kobayashi, NTT Corp. (Japan)

6891 OP Thermally stable multi-mode polymer optical waveguide fabricated by single-step photopatterning of fluorinated polyimide/epoxy hybrids [6891-24]

Y. Urano, N. Chen, Tokyo Institute of Technology (Japan); K. Nakano, K. Maeda, NEC Corp. (Japan); S. Ando, Tokyo Institute of Technology (Japan)

$68910 Q \quad$ Polysilane-based 3D waveguides for optical interconnects [6891-25]

K. Ogura, T. Oka, E. Watanabe, K. Aoi, H. Tsushima, Nippon Paint Co., Ltd. (Japan);

H. Okano, S. Suzuki, Hirose Electric Co., Ltd. (Japan); S. Hiramatsu, Mitsubishi Electric Corp. (Japan)

\section{SESSION 7 MISCELLANEOUS}

6891 OU Effect of ionizing radiation on infrared polarizers based on conductive polymer [6891-29] R. R. Boye, C. M. Washburn, Sandia National Labs. (USA); S. Samora, L\&M Technologies, Inc. (USA); S. A. Kemme, D. R. Wheeler, D. L. Buller, Sandia National Labs. (USA) 


\section{SESSION 8 ACTIVE MATERIALS}

$68910 Y \quad$ Near-infrared luminescent polymer waveguides and microlasers [6891-33]

M. Djiango, T. Kobayashi, W. J. Blau, Trinity College Dublin (Ireland); B. Cai, K. Komatsu,

T. Kaino, Tohoku Univ. (Japan)

$68910 Z$ Fabrication and evaluation of all-optical 1×2 Y-branch waveguide switch using photochromic amorphous molecular materials [6891-34]

Y. Narisawa, K. Komatsu, O. Sugihara, Tohoku Univ. (Japan); T. Tanino, H. Nakano, Osaka

Univ. (Japan); Y. Shirota, Fukui Univ. of Technology (Japan); T. Kaino, Tohoku Univ. (Japan)

\section{SESSION 9 NANOMATERIALS}

689112 Laser fabrication and crystallization of nano materials (Keynote Paper) [6891-37]

T. Sugiyama, Hamano Life Science Research Foundation (Japan) and Osaka Univ. (Japan); T. Asahi, K. Yuyama, H. Takeuchi, H.-G. Jeon, Osaka Univ. (Japan); Y. Hosokawa, H. Masuhara, Hamano Life Science Research Foundation (Japan) and Osaka Univ. (Japan)

689114 External field-induced orientation of DAST nanocrystals in the dispersion state and the fixation (Invited Paper) [6891-39]

H. Kasai, Tohoku Univ. (Japan) and PRESTO, Japan Science and Technology Agency (Japan); Y. Kaneko, T. Onodera, H. Nakanishi, H. Oikawa, Tohoku Univ. (Japan); S. Okada, Yamagata Univ. (Japan); S. Shimada, T. Kimura, H. Matsuda, National Institute of Advanced Industrial Science and Technology (Japan)

689116 Chemical etching of nanocomposite metal-semiconductor films monitored by Raman spectroscopy and surface probe microscopy [6891-45]

C. C. Perry, Oakwood College (USA); T. Brower, C. Zhang, Howard Univ. (USA); E. Waddell, Univ. of Alabama in Huntsville (USA); C. W. Bates, Jr., J. W. Mitchell, Howard Univ. (USA)

\section{POSTER SESSION}

689117 Characterization of polymer amplifier with large core fabricated using a dye vapor [6891-41]

H. Mochizuki, K. Murai, National Institute of Advanced Industrial Science and Technology (Japan)

6891 1A Efficiency improvement of flexible phosphorescent organic light emitting diode by inserting a buffer layer [6891-48]

Y. S. Tsai, S. H. Wang, F. S. Juang, S. Y. Su, National Formosa Univ. (Taiwan); M. H. Chang, Industrial Technology Research Institute (Taiwan); T. E. Hsieh, National Chiao-Tung Univ. (Taiwan); M. O. Liu, Industrial Technology Research Institute (Taiwan) 
Downloaded From: https://www.spiedigitallibrary.org/conference-proceedings-of-spie on 26 Apr 2023

Terms of Use: https://www.spiedigitallibrary.org/terms-of-use 


\title{
Conference Committee
}

\author{
Symposium Chair
}

Ali Adibi, Georgia Institute of Technology (USA)

Symposium Cochair

James G. Grote, Air Force Research Laboratory (USA)

Program Track Chair

James G. Grote, Air Force Research Laboratory (USA)

Conference Chairs

Robert L. Nelson, Air Force Research Laboratory (USA)

Francois Kajzar, Université d'Angers (France)

Toshikuni Kaino, Tohoku University (Japan)

Conference Cochair

Nakjoong Kim, Hanyang University (South Korea)

Program Committee

Chantal Andraud, Ecole Normale Supérieure de Lyon (France)

Werner J. Blau, The University of Dublin, Trinity College (Ireland)

Sophie Brasselet, Ecole Normale Supérieure de Cachan (France)

Christoph Bubeck, Max-Planck-Institut für Polymerforschung

(Germany)

Darnell E. Diggs, Air Force Research Laboratory (USA)

Alain F. Fort, Institut de Physique et Chimie des Matériaux de

Strasbourg (France)

James G. Grote, Air Force Research Laboratory (USA)

Makoto Hikita, NTT Advanced Technology Corporation (Japan)

F. Kenneth Hopkins, Air Force Research Laboratory (USA)

Alex K.-Y. Jen, University of Washington (USA)

Junji Kido, Yamagata University (Japan)

Jang-Joo Kim, Seoul National University (South Korea)

Isabelle N. Ledoux-Rak, Ecole Normale Supérieure de Cachan

(France)

Charles Y. C. Lee, Air Force Office of Scientific Research (USA)

Kwang-Sup Lee, Hannam University (South Korea) 
Misoon Mah, Air Force Research Laboratory (USA)

Seth R. Marder, Georgia Institute of Technology (USA)

Robert A. Norwood, College of Optical Sciences, The University of

Arizona (USA)

Jean-Michel Nunzi, Queens University (Canada)

Susanna Orlic, Technische Universität Berlin (Germany)

lleana Rãu, Politehnica University Bucharest (Romania)

Devanand K. Shenoy, Defense Advanced Research Projects Agency (USA)

Kenneth D. Singer, Case Western Reserve University (USA)

Don J. Smith, U.S. Air Force (USA)

Rebecca E. Taylor, Lockheed Martin Corporation (USA)

Toshiyuki Watanabe, Sumiden Opcom (Japan)

Jeong-Weon Wu, Ewha Womans University (South Korea)

Session Chairs

1 Multiphoton Absorption

Robert L. Nelson, Air Force Research Laboratory (USA)

2 Nonlinear Optics

lleana Rãu, Politehnica University Bucharest (Romania)

3 Materials

Francois Kajzar, Université d'Angers (France)

$4 \quad$ Applications

Toshikuni Kaino, Tohoku University (Japan)

5 Bio- and Nanomaterials

Kwang-Sup Lee, Hannam University (South Korea)

6 Optical Waveguide

Robert A. Norwood, College of Optical Sciences, The University of Arizona (USA)

$7 \quad$ Miscellaneous

Antoni Mitus, Wroclaw University of Technology (Poland)

8 Active Materials

James G. Grote, Air Force Research Laboratory (USA)

9 Nanomaterials

Christoph Bubeck, Max-Planck-Institut für Polymerforschung (Germany) 